\title{
Variables That Best Differentiate In-Patient Acute Stroke from Stroke-Mimics with Acute Neurological Deficits
}

\author{
P. Natteru, ${ }^{1}$ M. R. Mohebbi, ${ }^{2}$ P. George, ${ }^{3}$ D. Wisco, ${ }^{3}$ J. Gebel, ${ }^{4}$ and C. R. Newey ${ }^{1}$ \\ ${ }^{1}$ Department of Neurology, University of Missouri, Columbia, 5 Hospital Drive, CE 540, Columbia, MO 65211, USA \\ ${ }^{2}$ Department of Emergency Medicine, University of Missouri, Columbia, Columbia, MO, USA \\ ${ }^{3}$ Cleveland Clinic, 9500 Euclid Ave, Cleveland, OH 44195, USA \\ ${ }^{4}$ Akron General Hospital, 3562 Ridge Park Dr, Akron, OH 44333, USA
}

Correspondence should be addressed to C. R. Newey; neweyc@health.missouri.edu

Received 28 July 2016; Accepted 13 November 2016

Academic Editor: Tauheed Ishrat

Copyright (c) 2016 P. Natteru et al. This is an open access article distributed under the Creative Commons Attribution License, which permits unrestricted use, distribution, and reproduction in any medium, provided the original work is properly cited.

\begin{abstract}
Introduction. Strokes and stroke-mimics have been extensively studied in the emergency department setting. Although in-hospital strokes are less studied in comparison to strokes in the emergency department, they are a source of significant direct and indirect costs. Differentiating in-hospital strokes from stroke-mimics is important. Thus, our study aimed to identify variables that can differentiate in-hospital strokes from stroke-mimics. Methods. We present here a retrospective analysis of 93 patients over a oneyear period (2009 to 2010), who were evaluated for a concern of in-hospital strokes. Results. About two-thirds (57) of these patients were determined to have a stroke, and the remaining (36) were stroke-mimics. Patients with in-hospital strokes were more likely to be obese $(p=0.03)$, have been admitted to the cardiology service $(p=0.01)$, have atrial fibrillation $(p=0.03)$, have a weak hand or hemiparesis $(p=0.03)$, and have a prior history of stroke $(p=0.05)$, whereas, when the consults were called for "altered mental status" but no other deficits $(p<0.0001)$, it is likely a stroke-mimic. Conclusion. This study demonstrates that in-hospital strokes are a common occurrence, and knowing the variables can aid in their timely diagnosis and treatment.
\end{abstract}

\section{Introduction}

Stroke is the fifth leading cause of death in the United States (US) [1]. The economic and personal impact is huge considering the great direct and indirect costs, with a projection of US costs of ischemic stroke hitting $\$ 2.2$ trillion by 2050 [2]. Intravenous recombinant tissue plasminogen activator (IV rtPA) is approved for treating strokes in appropriate patients within 4.5 hours of stroke onset [3].

The clinical diagnosis of an acute stroke, however, can be a matter of dispute [4-6]. Typically, an acute stroke is considered in any patient who presents with sudden onset of neurological changes that can be localized to a vascular territory. However, other diagnoses, such as metabolic/infectious encephalopathy, seizure, syncope, peripheral neuropathy, space-occupying lesions, and migraines, may mimic a stroke, as observed in 22 to $31 \%$ of patients presenting to the emergency department (ED) with stroke-like symptoms [7, 8]. These may not be recognized until further diagnostic testing is available. In one of the studies by Goyal et al., treating the stroke-mimics (SM) with IV rt-PA led to an excess direct and indirect hospital cost of $\$ 257,975$ and $\$ 152,813$, respectively, and the median excess cost per admission was $\$ 5401$ [9].

Data for in-hospital strokes is, however, limited. This population represents up to $15 \%$ of first-time strokes [10]. Although in-hospital stroke-mimics have not been studied as extensively as those presenting to the ED, in-hospital strokes are often more severe and associated with a worse outcome [11]. These observations suggest the importance of recognizing in-hospital strokes and differentiating them from strokemimics, which can be difficult in comparison to out-ofhospital strokes.

At our institution, an in-patient acute stroke paging program has been established for over a decade (a.k.a. 2CLOT). It is an alerting system to the stroke fellow, resident, and staff. The purpose of this retrospective data analysis was to evaluate the in-patient stroke consults for the characteristics of strokes and stroke-mimics, that is, the timing, circumstances, and 
etiologies, with the ultimate goal of identifying differentiating variables that may allow for improved triaging.

\section{Methods}

This study is a retrospective data analysis of consecutive patients over a one-year period (2009 to 2010) at a large, tertiary-care referral center. The local institutional review board (IRB) approved the study.

2.1. Inclusion Criteria. The patient sample (18 years and older) included all consecutive acute stroke consults (or what our institution refers to as 2CLOTs), who were already admitted as an in-patient at the time of activation. Additionally, patients admitted to the in-house neurological floor and the neurological intensive care unit were excluded. The acute stroke consult was activated for urgent in-patient evaluations for any change in the neurological status from previous examinations. These consult activations could be from physicians, nurses, or ancillary staff.

2.2. Data Collection. Data was retrieved from the electronic medical records. The retrieved data included many variables like the initial National Institute of Health Stroke Scale (NIHSS) score, the circumstances of the brain attacks including time of onset, setting of onset (awakening from sleep, after operation, and sedation), time to presentation/evaluation, and examination at onset (blood pressure and heart rate, current atrial fibrillation, mental status, lateralizing features, neurological examination, and metabolic abnormalities). Historical data included prior medications, surgical history, past medical history (hypertension, ischemic heart disease, tobacco abuse, atrial fibrillation, diabetes mellitus, peripheral vascular disease, and prior stroke), stroke-mimic risk factor history like cognitive impairment, migraine, and epilepsy. Computed tomography (CT) and CT-angiography (CTA) of the head and vessels, magnetic resonance imaging (MRI) brain and magnetic resonance angiography (MRA) of the carotids and the circle of Willis, transcranial Doppler (TCD), electroencephalogram (EEG), and carotid ultrasounds (CUS) were the diagnostic data collected.

2.3. Determination of Stroke or Stroke-Mimic. The diagnoses of the patients were determined by the criteria used by Hand et al. [7]. The data were classified as a definite stroke/TIA, probable stroke/TIA, possible stroke/TIA, and definite nonstroke. A definite stroke is when history and examination are typical of a vascular event and there is supporting neuroimaging data. A definite TIA has resolution of symptoms within 24 hours and no neuroimaging sequela. A probable stroke was when clinical features suggest a vascular etiology, but neuroimaging does not support the claim, at the time of acquisition. Possible stroke had less convincing evidence of vascular etiology (e.g., encephalopathy) and another explanation is more reasonable. A definite nonstroke was when the clinical features did not suggest a vascular etiology, and supportive investigations convene to an alternate explanation (e.g., tumors). The data was then grouped as stroke (definite and probable stroke/TIA) or stroke-mimic (definite nonstroke and possible stroke/TIA). These diagnoses were determined by the initial assessment of the resident/fellow, staff, and then the final study reviewer. In case of a discrepancy, the diagnoses were reached by consensus. The kappa value calculated between the resident, fellow, staff, and the final study reviewer was moderate at 0.6 (63.4\% agreement).

2.4. Statistical Analysis. Differences between the two groups (stroke versus stroke-mimic) were assessed with descriptive statistics. Chi-square (categorical variables), $t$-test (continuous), and binary multiple logistic regression (multivariate analysis) were used to compare strokes versus stroke-mimics. Odds ratios (OR) were calculated between the two groups (stroke versus mimic) with 95\% confidence intervals for univariate analysis. SPSS (IBM Corporation, version 20.0, Armonk, NY) was used for statistical analyses.

\section{Results}

During the 12-month period, a total of 93 patients (average age $69.6 \pm 12.9$ years) were included in our study. After a panel review, 57 patients (mean age of 69.6 years, 57.9\% female) were determined as having a stroke (definite stroke/TIA or probable stroke/TIA). Thirty-six patients (mean age of 69.5 years, $50 \%$ female) were identified as having a stroke-mimic (possible stroke/TIA or definite nonstrokes). The majority of patients were discharged home or to rehabilitation (86\%). Baseline characteristics of the strokes and stroke-mimics are found in Table 1.

3.1. Circumstances of Acute Stroke Notifications. For all patients, the median NIHSS score was 10 (range of 0-41) with $34.0 \%$ having an NIHSS of $\leq 5$. Ten $(27.8 \%)$ of the stroke-mimics had NIHSS $\leq 5$ compared to $42.1 \%$ of stroke patients. This difference was nonsignificant. The exact time of onset was determined in all patients with the average time of neurological evaluation from last normal being $26.5 \pm$ 123.8 hours. The most common primary care team were cardiothoracic surgery (33\%), closely followed by cardiology (29\%). Eighteen patients (19.4\%) presented with symptoms first noticed upon awakening.

It was noted that stroke patients were significantly more likely to be obese $(p=0.03)$, had a prior history of stroke $(p=0.05)$ and atrial fibrillation at the time of onset or within 72 hours of the stroke activation $(p=0.03)$, and had been admitted to the cardiology service $(p=0.01)$. A total of fiftyeight patients were admitted to the cardiovascular service (cardiothoracic surgery and cardiology). Of those with atrial fibrillation at the time of onset or within 72 hours of the stroke, seventeen were on antiplatelet $(n=14)$ or therapeutic anticoagulation $(n=3)$. Nine patients were considered subtherapeutic on anticoagulation. Five patients were never on antiplatelet or anticoagulation. Two had antiplatelet medications withdrawn during hospitalization. Additionally, the subjective complaint of a weak hand ( $p=0.03$, OR 6.24) or objective hemiparesis ( $p=0.03$, OR 3.6) was more likely a stroke. In contrast, if a consult was for subjective altered mental status, it was significantly more likely a stroke-mimic $(p \leq 0.0001$; Tables 1 and 2).

There were no significant differences between the two groups in comparison of medications, laboratory values, 
TABLE 1: Patient characteristics of strokes and stroke-mimics.

\begin{tabular}{|c|c|c|c|c|c|c|}
\hline & \multicolumn{2}{|c|}{$\begin{array}{c}\text { All patients } \\
n=93\end{array}$} & \multicolumn{2}{|c|}{$\begin{array}{l}\text { Stroke patients only } \\
\qquad n=57\end{array}$} & \multicolumn{2}{|c|}{$\begin{array}{c}\text { Mimics only } \\
n=36\end{array}$} \\
\hline Age, $y$, mean $\pm S D$ & \multicolumn{2}{|c|}{$69.6 \pm 12.9$} & \multicolumn{2}{|c|}{$69.6 \pm 13.8$} & \multicolumn{2}{|c|}{$69.5 \pm 11.7$} \\
\hline Male, $n(\%)$ & 42 & 45.2 & 24 & 42.1 & 18 & 50.0 \\
\hline Hrs from onset, mean \pm SD & \multicolumn{2}{|c|}{$26.5 \pm 123.8$} & \multicolumn{2}{|c|}{$38.2 \pm 157.2$} & \multicolumn{2}{|c|}{$8.02 \pm 15.0$} \\
\hline \multicolumn{7}{|l|}{ Past history/stroke risk factors: } \\
\hline $\mathrm{HTN}, n(\%)$ & 64 & 68.8 & 37 & 64.9 & 27 & 75 \\
\hline Diabetes mellitus, $n(\%)$ & 23 & 24.7 & 13 & 22.8 & 10 & 27.8 \\
\hline Hypercholesterolemia, $n$ (\%) & 43 & 46.2 & 22 & 38.6 & 21 & 58.3 \\
\hline Atrial fibrillation, $n(\%)$ & 39 & 41.9 & 29 & 50.9 & 10 & $27.8^{*}$ \\
\hline CAD, $n(\%)$ & 48 & 51.6 & 28 & 49.1 & 20 & 55.6 \\
\hline Past tobacco abuse, $n(\%)$ & 44 & 47.3 & 24 & 42.1 & 20 & 55.6 \\
\hline Cancer, $n(\%)$ & 15 & 16.1 & 7 & 12.3 & 8 & 22.2 \\
\hline Prior stroke, $n(\%)$ & 19 & 20.4 & 8 & 14 & 11 & $30.6^{*}$ \\
\hline Prior heart surgery, $n(\%)$ & 38 & 40.9 & 24 & 42.1 & 14 & 38.9 \\
\hline Obesity, $n(\%)$ & 15 & 16.1 & 13 & 22.8 & 2 & $5.6^{*}$ \\
\hline Family history of stroke, $n(\%)$ & 17 & 18.3 & 10 & 17.5 & 7 & 19.4 \\
\hline \multicolumn{7}{|l|}{ Presentation: } \\
\hline Awoke from sleep, $n(\%)$ & 18 & 19.4 & 13 & 22.8 & 5 & 13.9 \\
\hline Coma, $n(\%)$ & 10 & 10.8 & 6 & 10.5 & 4 & 11.1 \\
\hline Altered mental status, $n(\%)$ & 33 & 35.3 & 12 & 21.1 & 21 & $58.3^{* *}$ \\
\hline Weak hand, $n(\%)$ & 15 & 16.1 & 13 & 22.8 & 2 & $5.6^{*}$ \\
\hline Hemiparesis, $n(\%)$ & 28 & 30.1 & 22 & 38.6 & 6 & $16.7^{*}$ \\
\hline AMET/CMET involved, $n(\%)$ & 6 & 6.45 & 4 & 7.02 & 2 & 6 \\
\hline 2CLOT activation by non-MD provider, $n(\%)$ & 59 & 63.4 & 37 & 64.9 & 22 & 61.1 \\
\hline Cardiology, $n(\%)$ & 27 & 29 & 22 & 38.6 & 5 & $13.9^{*}$ \\
\hline Cardiothoracic surgery service, $n(\%)$ & 31 & 33.3 & 19 & 33.3 & 12 & 33.3 \\
\hline \multicolumn{7}{|l|}{ Medication: } \\
\hline Antiplatelet, $n(\%)$ & 59 & 63.4 & 35 & 61.4 & 24 & 66.7 \\
\hline Anticoagulation, $n(\%)$ & 23 & 24.7 & 16 & 28.1 & 7 & 19.4 \\
\hline Statin, $n(\%)$ & 35 & 37.6 & 20 & 35.1 & 15 & 41.7 \\
\hline \multicolumn{7}{|l|}{ Examination: } \\
\hline $\mathrm{SBP}$, mean $\pm \mathrm{SD}$ & \multicolumn{2}{|c|}{$128 \pm 26.6$} & \multicolumn{2}{|c|}{$131 \pm 27.2$} & \multicolumn{2}{|c|}{$123 \pm 25.1$} \\
\hline Pulse, mean \pm SD & \multicolumn{2}{|c|}{$82.3 \pm 18.4$} & \multicolumn{2}{|c|}{$84.2 \pm 18.8$} & \multicolumn{2}{|c|}{$79.3 \pm 17.5$} \\
\hline Temperature (Celsius), mean \pm SD & \multicolumn{2}{|c|}{$36.5 \pm 0.72$} & \multicolumn{2}{|c|}{$36.6 \pm 0.75$} & & \\
\hline Baseline NIHSS, median (range) & 10 & $0-41$ & 10 & $0-41$ & 11.5 & $0-35$ \\
\hline $\mathrm{NIHSS} \leq 5, n(\%)$ & 34 & 36.6 & 24 & 42.1 & 10 & 27.8 \\
\hline MRS 0 to $2, n(\%)$ & 66 & 71 & 41 & 71.9 & 25 & 69.4 \\
\hline Laboratory/imaging: & & & & & & \\
\hline $\mathrm{WBC}, \mathrm{K} / \mathrm{uL}$, mean $\pm \mathrm{SD}$ & & & & & & \\
\hline Platelets, K/uL, mean \pm SD & & & & & & \\
\hline Sodium, $\mathrm{mmol} / \mathrm{L}$, mean $\pm \mathrm{SD}$ & & & & & & \\
\hline $\mathrm{BUN}, \mathrm{mg} / \mathrm{dL}$, mean $\pm \mathrm{SD}$ & & & & & & \\
\hline Creatinine, $\mathrm{mg} / \mathrm{dL}$, mean $\pm \mathrm{SD}$ & & & & & & \\
\hline Glucose, $\mathrm{mg} / \mathrm{dL}$, mean $\pm \mathrm{SD}$ & & & & & & \\
\hline Brain MRI, $n(\%)$ & 34 & 36.6 & 23 & 40.4 & 11 & 30.6 \\
\hline Discharged: & & & & & & \\
\hline Home, $n(\%)$ & 40 & 43 & 23 & 40.4 & 17 & 47.2 \\
\hline Rehabilitation, $n(\%)$ & 40 & 43 & 25 & 43.9 & 15 & 41.7 \\
\hline Death, $n(\%)$ & 13 & 14 & 9 & 15.8 & 4 & 11.1 \\
\hline
\end{tabular}

${ }^{*} p<0.05 ;{ }^{* *} p<0.0001$.

CAD, coronary artery disease; HTN, hypertension; 2CLOT, in-hospital stroke alerting system, AMET/CMET, in-hospital code blue team; NIHSS, NIH Stroke Scale; MRS, Modified Rankin Scale. 
TABLE 2: Multivariate analysis.

\begin{tabular}{lcc}
\hline Variables & OR & $p$ \\
\hline Hemiparesis & 3.6 & 0.042 \\
NIHSS item 1b & 0.46 & 0.17 \\
Normal mental status & 0.28 & 0.34 \\
Subjective complaint of weak hand & 6.24 & 0.042 \\
\hline
\end{tabular}

NIHSS, National Institute of Health Stroke Scale; OR, odds ratio.

TABLE 3: Stroke mechanisms.

\begin{tabular}{lcc}
\hline Stroke mechanisms & $n$ & $\%$ \\
\hline Cardioembolic & 41 & 71.9 \\
Large vessel & 8 & 14.0 \\
Small vessel & 2 & 3.5 \\
Other etiologies & 4 & 7.0 \\
Cryptogenic & 2 & 3.5 \\
\hline
\end{tabular}

n, number.

vitals, state of consciousness, past surgical history, and past medical history of hypertension, hypercholesterolemia, coronary artery disease, diabetes mellitus, past tobacco abuse, peripheral vascular disease, migraine, epilepsy, cancer, prior heart surgery, or a family history of stroke.

3.2. Etiologies of Strokes and Stroke-Mimics. The majority of strokes were found to be cardioembolic (71.9\%) compared to a large vessel occlusion (14.0\%) or small vessel occlusion (3.5\%; Table 3). The most common etiology of stroke-mimics was toxic/metabolic (38.9\%) of which medications (opioid/benzo) were the most common source (28.6\%; Table 4). Other common etiologies included seizure (22.2\%) and syncope $(13.9 \%)$. One consult was called for pain related to placement of a peripheral intravenous line.

3.3. Timing of the In-Hospital Strokes. Overall, the timing of evaluation from last known well was $436.8 \pm 768.1$ hours. Of the stroke patients, the majority $(n=29,50.9 \%)$ were assessed within three hours of last known well. The average times to neurology evaluation, to CT, and to treatment were $35 \mathrm{~min}$, $68 \mathrm{~min}$, and $237 \mathrm{~min}$, respectively (Table 5). The delay for inhospital strokes was in obtaining the CT and then initiating the treatment.

\section{Discussion}

An efficient stroke alerting system for in-patients is an advancement that is continuously evolving. The clinical assessment of acute strokes remains the standard and largely determines therapy. However, determining a stroke versus a stroke-mimic can be difficult. This study highlights that of the 93 patients who were suspected of having an acute stroke at our institution, the majority $(61.3 \%)$ were found to have a stroke with the remainder having a stroke-mimic.

Our findings are consistent with a study by Byrne et al., who reviewed 106 patients admitted to an in-patient stroke unit, and found that $78(73.5 \%)$ of the patients had strokes
TABLE 4: Etiologies of stroke-mimics.

\begin{tabular}{lcc}
\hline Stroke-mimics & $n$ & $\%$ \\
\hline Toxic/metabolic ${ }^{*}$ & 14 & 38.9 \\
Seizure & 8 & 22.2 \\
Syncope & 5 & 13.9 \\
Sepsis & 4 & 11.1 \\
Tumor & 1 & 2.8 \\
Peripheral neuropathy & 1 & 2.8 \\
Functional & 1 & 2.8 \\
Dementia & 1 & 2.8 \\
Spinal cord lesion & 1 & 2.8 \\
Vestibular & 0 & 0.0 \\
Migraine & 0 & 0.0 \\
\hline${ }^{*}$ Toxic metabolic causes & $n$ & $\%$ \\
\hline Medication (opioid/benzo) & 4 & 28.6 \\
Hyponatremia & 2 & 14.3 \\
Pulmonary distress & 2 & 14.3 \\
Hepatic failure & 2 & 14.3 \\
Hyperglycemia & 1 & 7.1 \\
Renal/hyperuricemia & 1 & 7.1 \\
Hyperkalemia & 1 & 7.1 \\
Attempting IV line & 1 & 7.1 \\
\hline
\end{tabular}

IV, intravenous; benzo, benzodiazepine; $n$, number.

TABLE 5: In-hospital stroke times.

\begin{tabular}{lcc}
\hline Treated in-hospital strokes & Minutes & StDev \\
\hline Stroke onset to neuro evaluation & 34.8 & 21.4 \\
Stroke onset to treatment & 236.6 & 65.1 \\
Stroke onset to CT & 67.6 & 27.7 \\
Neuro evaluation to CT & 32.8 & 10.4 \\
Neuro evaluation to needle & 213.8 & 83.7 \\
\hline
\end{tabular}

StDev, standard deviation; neuro, neurological; CT, computed tomography.

or TIAs, and the remaining were stroke-mimics (26.4\%) [5]. A similar study has reported the occurrence of in-hospital stroke-mimics to be $41 \%$ [12]. However, stroke-mimics may account for up to $63.4 \%$ of the acute stroke consults [13].

Recognition of acute strokes in a timely manner is a challenge [14]. Clinical scores to identify strokes have been created for the ED [15]. However, their utility in the inhospital patients with neurological changes is limited given the significant comorbidities. Thus, collaborative educational initiatives among physicians and nursing to improve quality and timeliness of acute stroke consults are necessary.

Additionally, our study showed which predictor variables can delineate in-hospital strokes from stroke-mimics. The most significant predictors for a stroke were subjective complaint of a weak hand, objective hemiparesis, points given on NIHSS $1 b$, a normal mental status, being admitted on the cardiology service, having atrial fibrillation at the time of onset or during hospitalization, previous stroke history, and obesity, which agrees with previous estimates $[12,16]$. On the contrary, when a consult was called for altered mental status, it was most likely a stroke-mimic. 
From our study, an interesting distinguishing predictor of stroke from a stroke-mimic was atrial fibrillation at the time of the stroke or within 72 hours of the stroke. This finding may be a reflection of an institutional bias. Our institution has an active cardiac center (i.e., cardiology and cardiothoracic surgery) where $62.4 \%$ of the stroke alerts were activated. However, it is consistent with previous observations that patients with in-hospital strokes were more likely to have atrial fibrillation than out-of-hospital strokes [10, 11, 16-18]. Additionally, cardioembolic strokes are much more common in the in-hospital stroke population compared to the community strokes $[11,12,19,20] .31 .0 \%$ of the atrial fibrillation patients in our study were subtherapeutic on their anticoagulation with an additional $6.9 \%$ having their antithrombotics discontinued during hospitalization for surgical procedures. Indeed, withdrawal of antithrombotics and subtherapeutic anticoagulation are risks for ischemic stroke [20]. Previous studies have demonstrated that patients with cardioembolic strokes from atrial fibrillation have poorer prognosis [21]. Thus, early resumption of antithrombotics and/or therapeutic anticoagulation is imperative.

Stroke alerts for altered levels of consciousness are significantly more likely to be a stroke-mimic. This is consistent with the findings of Libman et al. [18] and an editorial by Benbadis et al. [22]. An in-hospital stroke study by El Husseini and Goldstein found similar results. They identified 93 in-hospital acute stroke consults and found that the consults for altered mental status were significantly likely a stroke-mimic with the most common diagnosis for a stroke-mimic ultimately being metabolic and/or infectious [13]. The commonest etiologies of stroke-mimics from our study were toxic/metabolic (especially benzos, opioids), seizures, syncope, and sepsis, which agrees with previous studies $[8,23]$.

A recent study evaluated the use of red cell distribution width (RDW) as a marker to differentiate a stroke from stroke-mimics in young patients and demonstrated that the mean RDW values of young patients with stroke $(14.9 \pm 1.2)$ were significantly higher than patients with stroke-mimics (epilepsy or MS) $(13.3 \pm 1.2,13.4 \pm 0.6, p<0.0001$, resp.) [24].

In general, the in-hospital stroke patients may be less likely to receive IV rt-PA, secondary to the longer time to neuroimaging [25]. Additionally, thrombolytic treatment may reflect the fact that in-patients having a neurological change have more complex medical comorbidities that must be taken into account during the evaluation, transportation to the CT scanner, and administration of thrombolytic therapy. Future studies should focus on improving time to CT and time from CT to treatment in the in-hospital setting and then evaluate treatment. Other diagnostic testing such as MRI and/or EEG in distinguishing a stroke from a stroke-mimic should not come at the cost of time in patients awaiting IV tPA [15].

Lastly, $42.1 \%$ of our stroke patients had NIHSS $\leq 5$ compared to $27.8 \%$ of our stroke-mimics. This is an interesting observation since traditionally NIHSS correlates with stroke severity [26], and higher NIHSS scores are commonly associated with strokes [23]. Traditionally in-patient strokes have longer length of stay and worse prognosis [27]. However, in our study, stroke-mimics did not have a significant difference from the stroke population in discharged needs. Our data may represent the complexity of our patient population.

Several limitations are identified with the present study. Our study is a retrospective chart review of recognized strokes. It is possible that clinically silent or minor strokes may not have been recognized by medical personnel and, thus, would not be included in this study. Additionally, the majority of patients were admitted for cardiac intervention which biases the population to having cardioembolic strokes. An MRI of the brain was only available in $36.6 \%$ of the patients to confirm strokes. Lastly, we could not follow patients longitudinally to track outcomes.

\section{Conclusion}

In summary, the in-hospital stroke patients are a unique treatment group given their medical complexity which can pose challenges in acute stroke management. Differentiating stroke from stroke-mimic is crucial. Based on our study, a patient is more likely to have an acute ischemic stroke when presenting with a subjective complaint of a weak hand, objective hemiparesis, significant points on the NIHSS $1 \mathrm{~b}$, normal mental status, having atrial fibrillation at the time of onset or during hospitalization, past stroke history, and obesity.

\section{Competing Interests}

The authors have no financial interests to report.

\section{Authors' Contributions}

P. Natteru, M. Mohebbi, P. George, D. Wisco, J. Gebel, and C. R. Newey contributed equally to the writing of the case and formatting of the images.

\section{Acknowledgments}

The authors would like to thank Michelle Winfield, RN, and Julie McNeil, RN, for their assistance in identifying patients for this study.

\section{References}

[1] D. Mozzafarian, E. J. Benjamin, A. S. Go et al., "Heart disease and stroke statistics-2015 update: a report from the American Heart Association," Circulation, vol. 131, pp. e29-e322, 2015.

[2] D. L. Brown, B. Boden-Albala, K. M. Langa et al., "Projected costs of ischemic stroke in the United States," Neurology, vol. 67, no. 8, pp. 1390-1395, 2006.

[3] K. R. Lees, E. Bluhmki, R. von Kummer et al., "Time to treatment with intravenous alteplase and outcome in stroke: an updated pooled analysis of ECASS, ATLANTIS, NINDS, and EPITHET trials," The Lancet, vol. 375, no. 9727, pp. 1695-1703, 2010.

[4] G. Besson, C. Robert, M. Hommel, and J. Perret, "Is it clinically possible to distinguish nonhemorrhagic infarct from hemorrhagic stroke?" Stroke, vol. 26, no. 7, pp. 1205-1209, 1995.

[5] B. Byrne, P. O’Halloran, and C. Cardwell, "Accuracy of stroke diagnosis by registered nurses using the ROSIER tool compared 
to doctors using neurological assessment on a stroke unit: a prospective audit," International Journal of Nursing Studies, vol. 48, no. 8, pp. 979-985, 2011.

[6] P. A. O'Brien, D. Q. Ryder, and C. Twomey, "The role of computed tomography brain scan in the diagnosis of acute stroke in the elderly," Age and Ageing, vol. 16, no. 5, pp. 319-322, 1987.

[7] P. J. Hand, J. Kwan, R. I. Lindley, M. S. Dennis, and J. M. Wardlaw, "Distinguishing between stroke and mimic at the bedside: The Brain Attack Study," Stroke, vol. 37, no. 3, pp. 769775, 2006.

[8] W. O. Tobin, J. G. Hentz, B. J. Bobrow, and B. M. Demaerschalk, "Identification of stroke mimics in the emergency department setting," Journal of Brain Disease, vol. 1, pp. 19-22, 2009.

[9] N. Goyal, S. Male, A. Al Wafai, S. Bellamkonda, and R. Zand, "Cost burden of stroke mimics and transient ischemic attack after intravenous tissue plasminogen activator treatment," Journal of Stroke and Cerebrovascular Diseases, vol. 24, no. 4, pp. 828-833, 2015.

[10] R. E. Kelley and A. G. Kovacs, "Mechanism of in-hospital cerebral ischemia," Stroke, vol. 17, no. 3, pp. 430-433, 1986.

[11] M. U. Farooq, M. J. Reeves, J. Gargano, S. Wehner, S. Hickenbottom, and A. Majid, "In-hospital stroke in a statewide stroke registry," Cerebrovascular Diseases, vol. 25, no. 1-2, pp. 12-20, 2008.

[12] H. J. Park, H. J. Cho, Y.-D. Kim et al., "Comparison of the characteristics for in-hospital and out-of-hospital ischaemic strokes," European Journal of Neurology, vol. 16, no. 5, pp. 582$588,2009$.

[13] N. El Husseini and L. B. Goldstein, “'Code stroke': hospitalized versus emergency department patients," Journal of Stroke and Cerebrovascular Diseases, vol. 22, no. 4, pp. 345-348, 2013.

[14] M. J. Alberts, "Thrombolysis in acute ischemic stroke," Stroke, vol. 24, no. 6, pp. 911-912, 1993.

[15] W. N. Whiteley, J. M. Wardlaw, M. S. Dennis, and P. A. G. Sandercock, "Clinical scores for the identification of stroke and transient ischaemic attack in the emergency department: a cross-sectional study," Journal of Neurology, Neurosurgery and Psychiatry, vol. 82, no. 9, pp. 1006-1010, 2011.

[16] J. G. Merino, M. Luby, R. T. Benson et al., "Predictors of acute stroke mimics in 8187 patients referred to a stroke service," Journal of Stroke \& Cerebrovascular Diseases, vol. 22, no. 8, pp. e397-e403, 2013.

[17] K. Kimura, K. Minematsu, and T. Yamaguchi, "Characteristics of in-hospital onset ischemic stroke," European Neurology, vol. 55, no. 3, pp. 155-159, 2006.

[18] R. B. Libman, E. Wirkowski, J. Alvir, and T. H. Rao, "Conditions that mimic stroke in the emergency department. Implications for acute stroke trials," Archives of Neurology, vol. 52, no. 11, pp. 1119-1122, 1995.

[19] J. Masjuan and R. Vera, "In-hospital stroke," European Journal of Neurology, vol. 16, no. 5, pp. 549-550, 2009.

[20] R. Vera, A. Lago, B. Fuentes et al., "In-hospital ischemic strokes in patients admitted to Cardiology and Cardiac Surgery departments. Multi-centre registry," Medicina Clinica, vol. 137, no. 11, pp. 479-483, 2011.

[21] C. Steger, A. Pratter, M. Martinek-Bregel et al., "Stroke patients with atrial fibrillation have a worse prognosis than patients without: data from the Austrian Stroke registry," European Heart Journal, vol. 25, no. 19, pp. 1734-1740, 2004.

[22] S. R. Benbadis, R. B. Libman, and E. Wirkowski, "Conditions that mimic stroke," Archives of Neurology, vol. 53, no. 5, pp. 404412, 1996.
[23] V. Quenardelle, V. Lauer-Ober, I. Zinchenko et al., "Stroke mimics in a stroke care pathway based on MRI screening," Cerebrovascular Diseases, vol. 42, no. 3-4, pp. 205-212, 2016.

[24] R. Demir, M. Saritemur, O. Atis et al., "Can we distinguish stroke and stroke mimics via red cell distribution width in young patients?" Archives of Medical Science, vol. 11, no. 5, pp. 958-963, 2015.

[25] A. P. Saltman, F. L. Silver, J. Fang, M. Stamplecoski, and M. K. Kapral, "Care and outcomes of patients with in-hospital stroke," JAMA Neurology, vol. 72, no. 7, pp. 749-755, 2015.

[26] D. C. Tong, M. A. Yenari, G. W. Albers, M. O’Brien, M. P. Marks, and M. E. Moseley, "Correlation of perfusion- and diffusionweighted MRI with NIHSS score in acute $(<6.5$ hour) ischemic stroke," Neurology, vol. 50, no. 4, pp. 864-869, 1998.

[27] A. Bhalla, N. Smeeton, A. G. Rudd, P. Heuschmann, and C. D. A. Wolfe, "A comparison of characteristics and resource use between in-hospital and admitted patients with stroke," Journal of Stroke and Cerebrovascular Diseases, vol. 19, no. 5, pp. 357$363,2010$. 


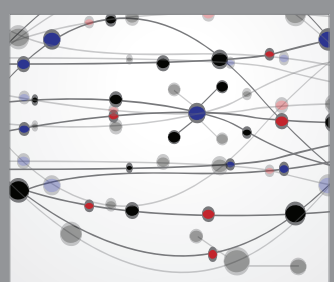

The Scientific World Journal
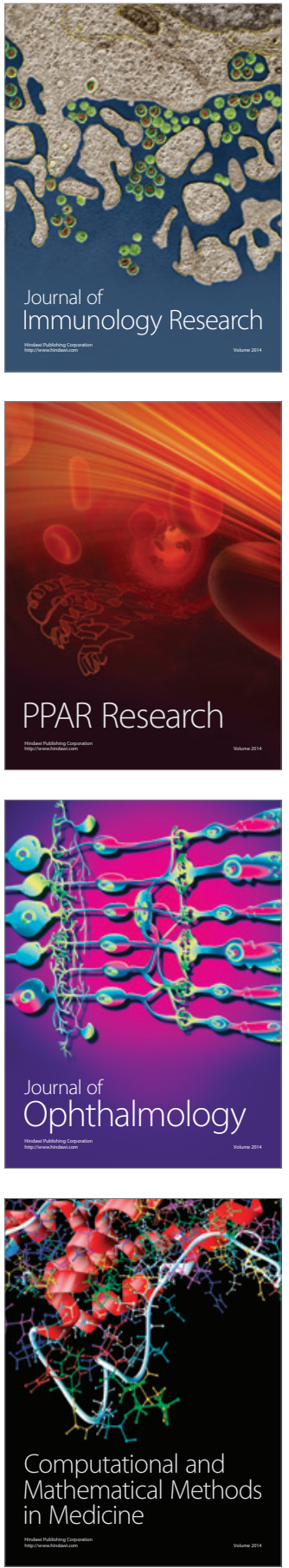

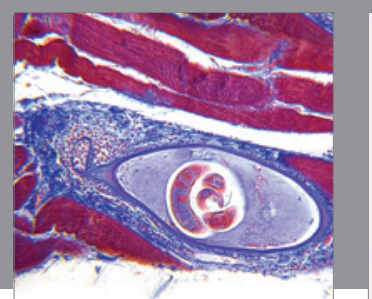

Gastroenterology Research and Practice

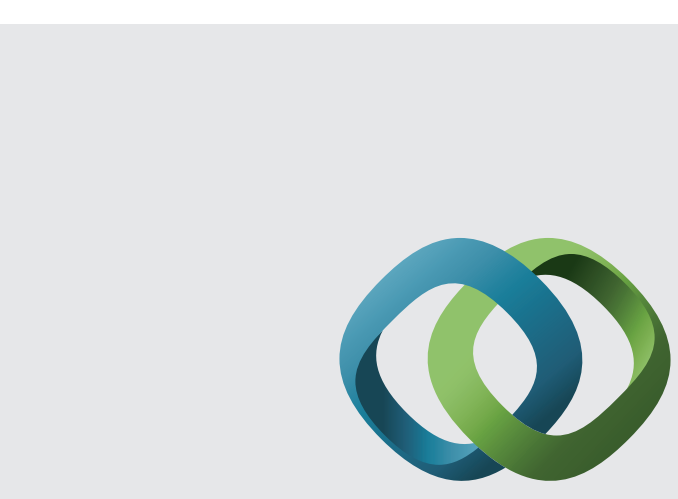

\section{Hindawi}

Submit your manuscripts at

http://www.hindawi.com
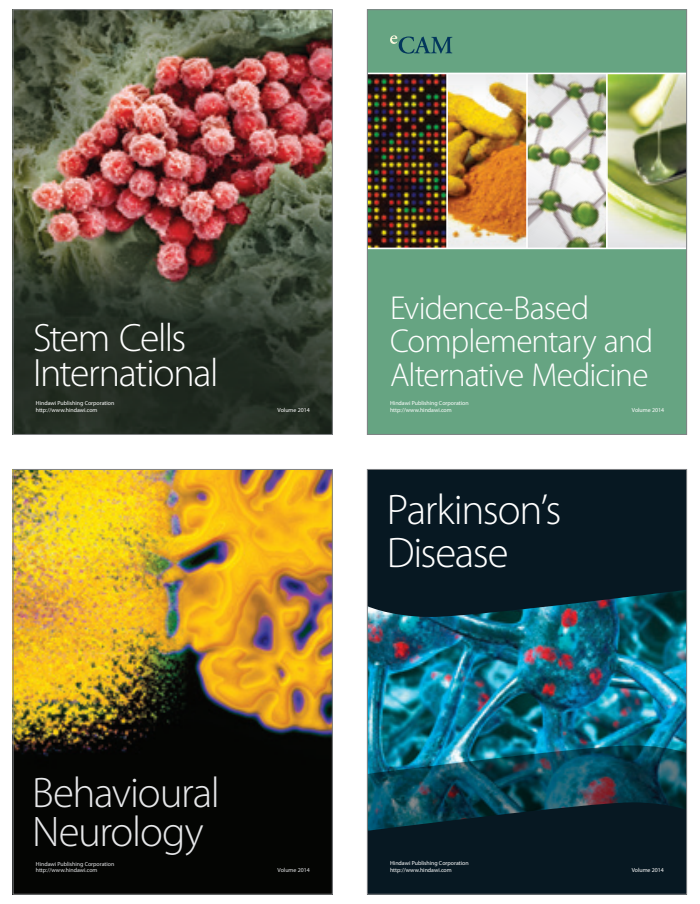
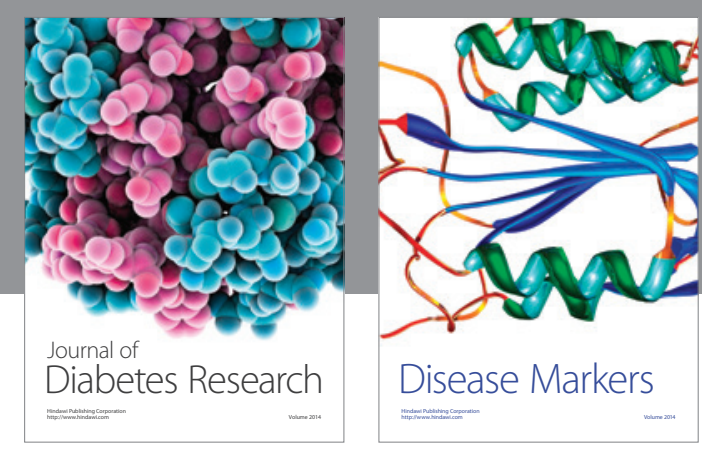

Disease Markers
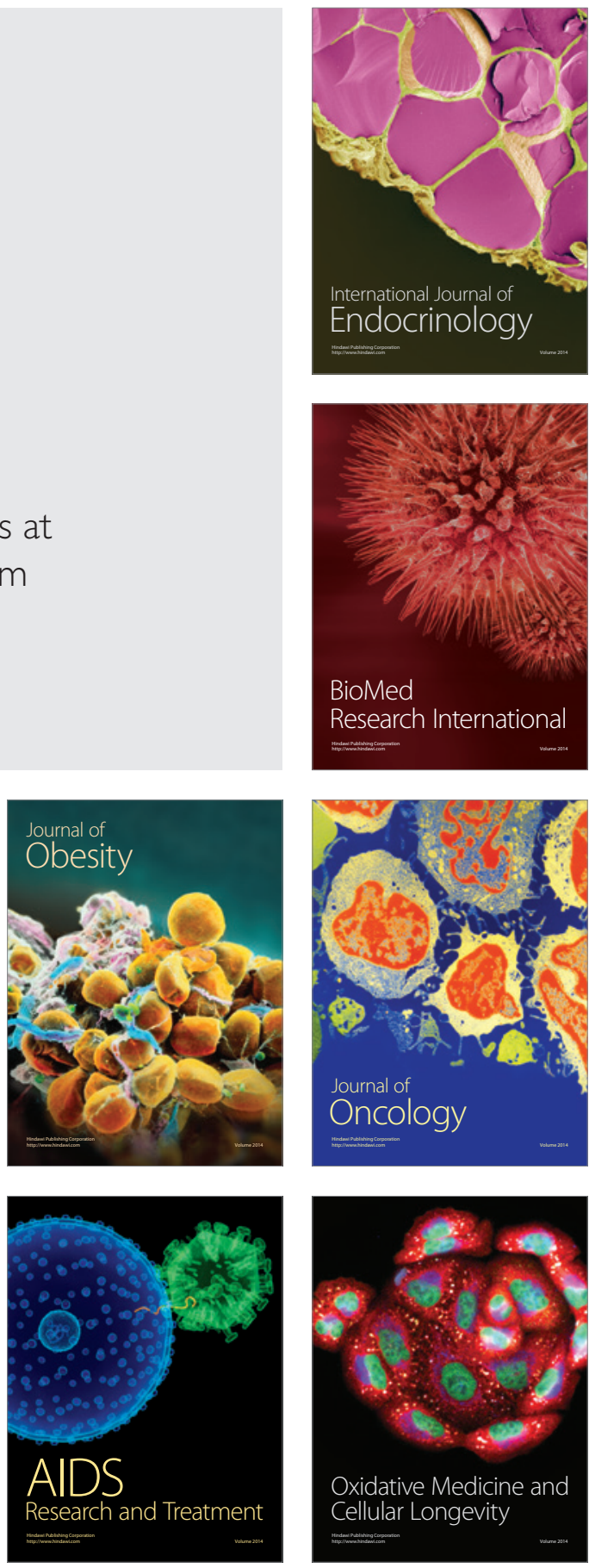